The Chinese Society of Theoretical and Applied Mechanics

Chinese Journal of Mechanics Press, Beijing, China

Allerton Press, INC., New York, U.S.A.

\title{
THE SCATTERING OF GENERAL SH PLANE WAVE BY INTERFACE CRACK BETWEEN TWO DISSIMILAR VISCOELASTIC BODIES
}

\author{
Wei Peijun (魏培君) Zhang Shuangyin (张双寅) Wu Yongli (吴永礼) \\ (Laboratory for Nonlinear Mechanics of Continuous Media, Institute of Mechanics, Chinese \\ Academy of Science, Beijing 100080, China) \\ Robert K.Y. Li \\ (Department of Physics and Materials Science, City University of Hong Kong, China)
}

\begin{abstract}
The scattering of general SH plane wave by an interface crack between two dissimilar viscoelastic bodies is studied and the dynamic stress intensity factor at the crack-tip is computed. The scattering problem can be decomposed into two problems: one is the reflection and refraction problem of general $\mathrm{SH}$ plane waves at perfect interface (with no crack); another is the scattering problem due to the existence of crack. For the first problem, the viscoelastic wave equation, displacement and stress continuity conditions across the interface are used to obtain the shear stress distribution at the interface. For the second problem, the integral transformation method is used to reduce the scattering problem into dual integral equations. Then, the dual integral equations are transformed into the Cauchy singular integral equation of first kind by introduction of the crack dislocation density function. Finally, the singular integral equation is solved by Kurtz's piecewise continuous function method. As a consequence, the crack opening displacement and dynamic stress intensity factor are obtained. At the end of the paper, a numerical example is given. The effects of incident angle, incident frequency and viscoelastic material parameters are analyzed. It is found that there is a frequency region for viscoelastic material within which the viscoelastic effects cannot be ignored.
\end{abstract}

KEY WORDS: viscoelasticity, interface crack, general plane wave, integral transformation, singular integral equations

\section{INTRODUCTION}

The scattering problem of interface cracks is of importance in theoretical analysis and engineering applications. It is an important aspect of dynamic fracture mechanics of interfaces, and can provide necessary information for the techniques of nondestructive evaluation (NDE). The problem can be separated into two problems: the first is the reflection and refraction problem at a perfect interface; the second is the scattering problem due to the existence of a crack.

Received 29 December 1998

* This work was supported by the National Natural Science Foundation of China (No.19772064) and by the project of CAS KJ $951-1-20$ 
For linear elastic materials, the reflection and refraction of elastic wave have been studied widely. Miklowitz ${ }^{[1]}$ and Achenbach ${ }^{[2]}$ both gave detailed discussions. The scattering problem of interface cracks was studied firstly by Srivastava ${ }^{[3,4]}$ on Griffith and penny-shaped cracks. Yang \& Body ${ }^{[5]}$ considered the scattering of an elastic wave at the interface crack in a layered half-space. The scattering of SH elastic waves at the far field from a Griffith interface crack was investigated by Bostrom ${ }^{[6]}$. The scatterings of $P$ and $S$ waves by single or multiple interface crack in layered medium were studied by $\mathrm{Ma}$ et al. ${ }^{[7]}$ and Zhang et al. ${ }^{[8]}$. $\mathrm{Qu}{ }^{[9,10]}$ studied the scattering at the near field and far field of a Griffith interface crack by using singular integral equation.

For viscoelastic materials, it is the viscoelastic wave that propagates. There are three important characteristics in which viscoelastic wave is different from elastic wave: 1) attenuation, 2) dispersion, and 3) movement coupling (i.e. the movement track of mass point is elliptical for $\mathrm{P}$ and $\mathrm{S}$ waves). These characteristics require the introduction of complex wave number and complex wave speed, which adds difficulties for the analysis. The studies of reflection and refraction of general plane wave dated back to the sixties when Lockett ${ }^{[11]}$ firstly investigated the reflection and refraction of $P$ and $S$ waves at free boundary and interface. It was found that reflection and refraction wave would be a fan-shaped section for compound incident waves. The investigation by Cooper ${ }^{[12,13]}$ on the same problem has identified the phase shift phenomena between reflection or refraction wave and incident wave. Schoenberg ${ }^{[14]}$ studied the reflection and refraction of SH wave and associated energy flow. Borchert ${ }^{[15]}$ studied the reflection and refraction of $P$ and $S$ wave and critical angle for incident wave. But the investigations of the scattering problem of viscoelastic wave by crack are rare. Only Georgiadis ${ }^{[16,17]}$ studied the dynamic response due to a sudden load for Griffith and penny-shaped cracks in homogenous viscoelastic bodies.

In this paper, the scattering problem of general SH plane wave due to an interface crack between two dissimilar viscoelastic bodies are considered, and the numerical results are obtained for the case of a Standard Linear Solid. The numerical study reveals an interesting feature: there is a frequency influence region existing for viscoelastic materials within which the viscoelastic effects cannot be ignored. In addition, the method in this paper can be used to study the scattering of $\mathrm{P}$ or $\mathrm{SV}$ waves.

\section{FORMULATION OF THE PROBLEM}

We consider a Griffith interface crack of length $2 a$ between two dissimilar, homogenous, isotropic, and linear viscoelastic solids of half-spaces. A Cartesian coordinate system is assumed in such a way that the $x$-axis is along the crack direction and the $y$-axis is perpendicular to crack direction, as shown in Fig.1. The crack is infinite wide in $z$-direction. Densities of the two materials are denoted by $\rho_{1}, \rho_{2}$ and shear relaxation moduli by $G_{1}(t), G_{2}(t)$, respectively. Standard linear solid model is used for the viscoelastic materials, where relaxation modulus can be written as

$$
\begin{array}{r}
G_{i}(t)=\mu_{\infty i}\left[1+f_{i} \exp \left(-\frac{t}{\tau_{i}}\right)\right] \\
(i=1,2)
\end{array}
$$

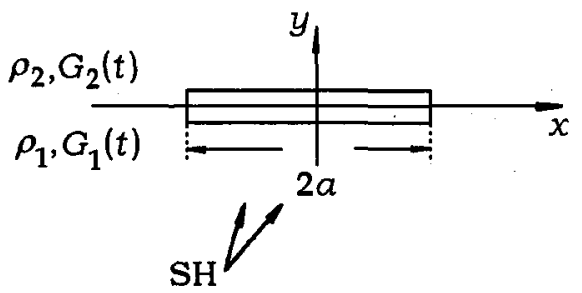

Fig.1 General SH plane incident wave on the interface crack between two dissimilar viscoelastic bodies 
in which $f_{i}=\frac{\mu_{0 i}}{\mu_{\infty i}}-1, \mu_{0}$ and $\mu_{\infty}$ are the instantaneous shear modulus and steady state shear modulus, respectively, and $\tau_{i}$ is the relaxation time of the viscoelastic materials.

Consider the case when there is an oblique incident general SH plane wave travelling from infinity in the viscoelastic solid. When it reaches the interface, reflection and refraction waves will be generated. The total displacement field can be written as

$$
U= \begin{cases}U_{1}^{\mathrm{i}}+U_{1}^{\mathrm{r}}+U_{1}^{\mathrm{s}} & y<0 \\ U_{2}^{\mathrm{t}}+U_{2}^{\mathrm{s}} & y>0\end{cases}
$$

where, $U_{1}^{\mathrm{i}}, U_{1}^{\mathrm{r}}$ and $U_{2}^{\mathrm{t}}$ are the incident, reflection and refraction displacement fields, respectively for a perfect interface, i.e. the one with no crack. $U_{1}^{\text {s }}$ and $U_{2}^{\text {s }}$ are the scattering displacement field due to the existence of a crack.

The boundary conditions of the problem are given as

$$
\begin{array}{ll}
\tau_{y z}(x, 0, t)=0 & |x|<a \\
\tau_{y z}\left(x, 0^{+}, t\right)=\tau_{y z}\left(x, 0^{-}, t\right) & -\infty \leq x \leq \infty \\
U\left(x, 0^{+}, t\right)=U\left(x, 0^{-}, t\right) & |x|>a \\
U(x, y, t)=0 & x, y \rightarrow \infty
\end{array}
$$

\section{REFLECTION AND REFRACTION OF GENERAL SH PLANE WAVE AT PERFECT INTERFACE}

The incident general SH plane wave can be written in the follow forms

$$
U_{1}^{\mathrm{i}}=w_{1}^{\mathrm{i}} \exp \left(\mathrm{i} P^{\mathrm{i}} \cdot r\right) \exp (\mathrm{i} \omega t)=w_{1}^{\mathrm{i}} \exp \left[\mathrm{i}\left(P_{x}^{\mathrm{i}} \cdot x+P_{y}^{\mathrm{i}} \cdot y\right)\right] \exp (\mathrm{i} \omega t)
$$

where, $w^{\mathrm{i}}$ is the complex amplitude in $z$ direction; $P_{x}^{\mathrm{i}}, P_{y}^{\mathrm{i}}$ are respectively the complex projection of complex vector $\boldsymbol{P}^{\mathrm{i}}$ along $x, y$ directions; $\boldsymbol{r}=\boldsymbol{x} \cdot \boldsymbol{x}+\boldsymbol{y} \cdot \boldsymbol{y}$ is the position vector.

In the case of SH incident wave, the reflection wave and refraction wave are the SH wave. Similarly, the reflection and refraction waves can be written respectively as

$$
\begin{aligned}
& U_{1}^{\mathrm{r}}=w_{1}^{\mathrm{r}} \exp \left(\mathrm{i} \boldsymbol{P}^{r} \cdot r\right) \exp (\mathrm{i} \omega t)=w_{1}^{\mathrm{r}} \exp \left[\mathrm{i}\left(P_{x}^{\mathrm{r}} \cdot x+P_{y}^{\mathrm{r}} \cdot y\right)\right] \exp (\mathrm{i} \omega t) \\
& U_{2}^{\mathrm{t}}=w_{2}^{\mathrm{t}} \exp \left(\mathrm{i} \boldsymbol{P}^{\mathrm{t}} \cdot \boldsymbol{r}\right) \exp (\mathrm{i} \omega t)=w_{2}^{\mathrm{t}} \exp \left[\mathrm{i}\left(P_{x}^{\mathrm{t}} \cdot x+P_{y}^{\mathrm{t}} \cdot y\right)\right] \exp (\mathrm{i} \omega t)
\end{aligned}
$$

For convenience, the common factor $\exp (\mathrm{i} \omega t)$ will be omitted hereafter.

All the displacement fields mentioned above satisfied the viscoelastic wave equation without exception, in which a complex wave number is included.

$$
\nabla^{2} U^{k}+k_{s}^{2} U^{k}=0 \quad \text { the superscript } k=\mathrm{i}, \mathrm{r}, \mathrm{t}
$$

where $k_{s}^{2}=\frac{\omega^{2} \rho}{\mu(\omega)}$ is the complex wave number; $\mu(\omega)=\mu_{0}+i \omega \widetilde{G}(\omega)$ is the complex modulus; and $\widetilde{G}(\omega)=\{F[G(t)], t \rightarrow \omega\}$ is the Fourier transformation of the shear relaxation modulus $G(t)$. 
Interface boundary conditions require

$$
\begin{array}{ll}
U_{1}^{\mathrm{i}}+U_{1}^{\mathrm{r}}=U_{2}^{\mathrm{t}} & y=0 \\
\mu_{1}(\omega) \frac{\partial}{\partial y}\left[U_{1}^{\mathrm{i}}+U_{1}^{\mathrm{r}}\right]=\mu_{2}(\omega) \frac{\partial}{\partial y} U_{2}^{\mathrm{t}} & y=0
\end{array}
$$

It follows from $(10) \sim(12)$ that reflection factor, refraction factors and interface shear stress are

$$
\begin{aligned}
& \beta_{r}=\frac{w_{1}^{\mathrm{r}}}{w_{1}^{\mathrm{i}}}=\frac{\mu_{1}(\omega) P_{y}^{\mathrm{i}}-\mu_{2}(\omega) P_{y}^{\mathrm{t}}}{\mu_{2}(\omega) P_{y}^{\mathrm{t}}+\mu_{1}(\omega) P_{y}^{\mathrm{i}}} \\
& \beta_{t}=\frac{w_{2}^{\mathrm{t}}}{w_{1}^{\mathrm{i}}}=\frac{2 \mu_{1}(\omega) P_{y}^{\mathrm{i}}}{\mu_{2}(\omega) P_{y}^{\mathrm{t}}+\mu_{1}(\omega) P_{y}^{\mathrm{i}}} \\
& \tau_{y z}^{\mathrm{t}}(x, 0, \omega)=\mu_{2}(\omega) \frac{\partial}{\partial y}\left(U_{2}^{\mathrm{t}}\right)=\mu_{2}(\omega) w^{\mathrm{i}} \beta_{t}\left(\mathrm{i} P_{y}^{\mathrm{t}}\right) \cdot \exp \left(\mathrm{i} P_{x}^{\mathrm{i}} \cdot x\right)
\end{aligned}
$$

\section{THE DERIVATION AND NUMERICAL SOLUTION OF SINGULAR INTEGRAL EQUATION FOR SCATTERING FIELD}

\subsection{Dual Integral Equations of Crack Opening Displacement (COD)}

The COD is defined as

$$
\operatorname{COD}=U_{2}^{\mathrm{s}}\left(x, 0^{+}, \omega\right)-U_{1}^{\mathrm{s}}\left(x, 0^{-}, \omega\right)= \begin{cases}\Delta w(x, \omega) & |x|<a \\ 0 & |x|>a\end{cases}
$$

The scattering displacement field $U^{s}(x, y)$ satisfies the viscoelastic wave equation (10) and radiation condition (6). By using the Fourier integral transformation, we can get scattering displacement field in the transformation domain, i.e. $\widetilde{U}^{\mathrm{s}}(s, y)$. By employing the interface displacement continuous conditions outside of crack and stress boundary conditions on crack surface, the dual integral equations about the unknown function $\Delta \widetilde{w}(s, \omega)$ were constructed

$$
\left.\begin{array}{ll}
F^{-1}[\Delta \tilde{w}]=0 & |x|>a \\
F^{-1}\left[\mu_{1}(\omega) \frac{\beta}{\beta+1} \sqrt{k_{s 1}^{2}-s^{2}} \Delta \tilde{w}\right]=-\tau_{y z}^{\mathrm{t}}(s, 0, \omega) & |x|<a
\end{array}\right\}
$$

where

$$
\beta(s, \omega)=\frac{\mu_{2}(\omega) \sqrt{k_{s 2}^{2}-s^{2}}}{\mu_{1}(\omega) \sqrt{k_{s 1}^{2}-s^{2}}}
$$

$\Delta \tilde{w}(s, \omega)=F[\Delta w(x, \omega)], F[]$ and $F^{-1}[]$ denote the Fourier transformation and inverse Fourier transformation, respectively.

\subsection{Singular Integral Equation of Crack Dislocation Density}

Crack dislocation density function is defined as

$$
\Phi(x, \omega)=\frac{\partial}{\partial x} \Delta w(x, \omega)
$$


Applying the Fourier integral transformation on $x$, Eq.(19) becomes

$$
\widetilde{\Phi}(s, \omega)=(\mathrm{i} s) \Delta \widetilde{w}(s, \omega)
$$

Substituting Eq.(20) into the first equation of Eqs.(17) yields

$$
\int_{-a}^{a} \Phi(x, \omega) \mathrm{d} x=0
$$

Substituting Eq.(20) into the second equation of Eqs.(17) yields

$$
\begin{gathered}
-\frac{1}{2 \pi \mathrm{i}} \int_{-\infty}^{\infty} \mu_{1}(\omega) \frac{\beta}{\beta+1} \sqrt{k_{s 1}^{2}-s^{2}} \frac{1}{s}\left[\int_{-a}^{a} \Phi(u) \mathrm{e}^{\mathrm{i} s u} \mathrm{~d} u\right] \mathrm{e}^{-\mathrm{i} s x} \mathrm{~d} s= \\
-\tau_{y z}^{\mathrm{t}}(x, 0, \omega) \quad|x|<a
\end{gathered}
$$

Considering

$$
\lim _{s \rightarrow \infty} \mu_{1}(\omega) \frac{\beta}{\beta+1} \sqrt{k_{s 1}^{2}-s^{2}} \frac{1}{s}=\frac{\mu_{1}(\omega) \mu_{2}(\omega)}{\mu_{1}(\omega)+\mu_{2}(\omega)} \operatorname{sign}(s)
$$

and using Riemman-Lebesgue lemma ${ }^{[18]}$

$$
\int_{-\infty}^{\infty} \mathrm{e}^{\mathrm{i} s(u-x)} \operatorname{sign}(s) \mathrm{d} s=\frac{2 \mathrm{i}}{u-x}
$$

we can reduce Eq.(22) into

$$
\frac{\mu_{1}(\omega) \mu_{2}(\omega)}{\mu_{1}(\omega)+\mu_{2}(\omega)} \frac{1}{\pi} \int_{-a}^{a} \frac{\Phi(u)}{u-x} \mathrm{~d} u+\int_{-a}^{a} K(u, \omega) \Phi(u, \omega) \mathrm{d} u=-\tau_{y z}^{t}(x, 0, \omega) \quad|x|<a
$$

where

$$
K(u, \omega)=-\frac{1}{2 \pi i} \int_{-\infty}^{\infty}\left[\mu_{1}(\omega) \frac{\beta}{\beta+1} \sqrt{k_{s 1}^{2}-s^{2}} \frac{1}{s}-\frac{\mu_{1}(\omega) \mu_{2}(\omega)}{\mu_{1}(\omega)+\mu_{2}(\omega)} \operatorname{sign}(s)\right] e^{-i s(u-x)} \mathrm{d} s
$$

This is the first kind of Cauchy singular integral equation (SIE).

\subsection{Numerical Resolution of Singular Integral Equation}

According to the theory of singular integral equation ${ }^{[19]}$, the dislocation density function can be written as

$$
\Phi(x, \omega)=\varphi(x, \omega) W(x)
$$

where the fundamental solution $W(x)=(a-x)^{1 / 2}(a+x)^{1 / 2}$ reflects the singularity of dislocation density function at the crack-tip. The real singular index implies that there is no oscillation singularity for interface cracks under anti-plane shear loading.

Because of the complexity of the integral kernel $K(u, \omega)$, formula (25) can only be solved by numerical methods. There are mainly two numerical methods that can be used. One is based on the Jacobi or Chebyshev orthogonal polynomials developed by Erdogan [20]. Another is based on the piecewise continuous polynomial developed by Kurtz ${ }^{[21]}$. The first method decomposes the unknown function into a series from an overall view

$$
\varphi(x)=\sum_{0}^{N} a_{n} P_{n}^{(\alpha, \beta)}(x)=\sum_{0}^{N} a_{n} T_{n}(x) \quad(\text { when } \alpha=\beta=1 / 2)
$$


and transforms the singular integral equation about $\varphi(x)$ into algebraic equations about the factors $a_{n}$. The second method decomposes the unknown function from a local view

$$
\varphi(x)=\sum_{1}^{N} \varphi_{i}(x)=\sum_{i=1}^{N} \sum_{j=1}^{n} \lambda_{i j}(x) \varphi_{i j}
$$

where $\varphi_{i}(x)=\sum_{j=1}^{n} \lambda_{i j}(x) \varphi_{i j}$ is an interpolation polynomial of order $n-1$ in the interval $\left[x_{i}, x_{i+1}\right]$, and transforms the singular integral equation about $\varphi(x)$ into algebraic equations about the collection point functions $\varphi_{i j}$.

In this paper, the piecewise continuous polynomial method is used, and the Lagrange interpolation polynomial of second order is used for $\varphi_{i}(x)$. The detailed process of the method will not be repeated here. After the crack dislocation density $\Phi(x)$ is obtained, we can get

$$
\begin{aligned}
& \Delta w(x, \omega)=\int_{-a}^{x} \Phi(x, \omega) \mathrm{d} x=\int_{-a}^{x} \varphi(x, \omega) W(x) \mathrm{d} x \\
& \tau_{y z}(x, 0, \omega)=-\frac{\mu_{1}(\omega) \mu_{2}(\omega)}{\mu_{1}(\omega)+\mu_{2}(\omega)} \frac{1}{\pi} \int_{-a}^{a} \frac{\Phi(u, \omega)}{u-x} \mathrm{~d} u+\int_{-a}^{a} K(u, \omega) \Phi(u, \omega) \mathrm{d} u \quad|x| \geq a \\
& K_{\mathrm{III}}=\lim _{x \rightarrow a} \sqrt{2 \pi(x-a)} \tau_{y z}(x, 0, \omega)
\end{aligned}
$$

\section{NUMERICAL EXAMPLE}

One example is given here to illustrate the use of the method aforementioned. The viscoelastic material parameters used in the numerical example are listed in Table 1 . The parameters of the incident wave are $w^{\mathrm{i}}=1+\mathrm{i}$. Obviously, as response to the incident wave, the crack opening displacement COD and the stress intensity factor $K_{\text {III }}$ are all functions of the incident angle $\theta^{i}$ and incident frequency $\omega$. In order to show sufficiently the effects of the incident wave, numerical calculations are carried out for various $\theta^{i}$ and $\omega$ values and the results were shown as $3 \mathrm{D}$ contour plots.

Table 1

\begin{tabular}{lcccc}
\hline & $\mu_{0} / \mathrm{MNm}^{-2}$ & $\mu_{\infty} / \mathrm{MNm}^{-2}$ & $\tau / \mathrm{s}$ & $\rho / \mathrm{kgm}^{-3}$ \\
\hline viscoelastic material 1 & $0.127 \mathrm{E} 10$ & $0.634 \mathrm{E} 9$ & 20.0 & 1200 \\
viscoelastic material 2 & $0.634 \mathrm{E} 9$ & $0.254 \mathrm{E} 9$ & 20.0 & 1200 \\
\hline
\end{tabular}

Figure 3 shows the variation of the complex modulus $\mu(\omega)$ with different incident frequencies $\omega$. It can be seen that the imaginary part of complex modulus $\mu(\omega)$ approaches zero for large and small $\omega$, and it has significant value only in a narrow frequency region. The region can be called the frequency influence region. For the viscoelastic parameters employed in this paper, this region is roughly defined as

$$
\begin{aligned}
& 10^{-3}<\text { frequency }(\omega)<10^{0} \\
& 2 \pi<\text { period }(T)<2 \pi \times 10^{3} \\
& 2 \pi \times \sqrt{\mu_{0} / \rho}<\text { wave length }(\lambda)<2 \pi \times 10^{3} \times \sqrt{\mu_{\infty} / \rho}
\end{aligned}
$$




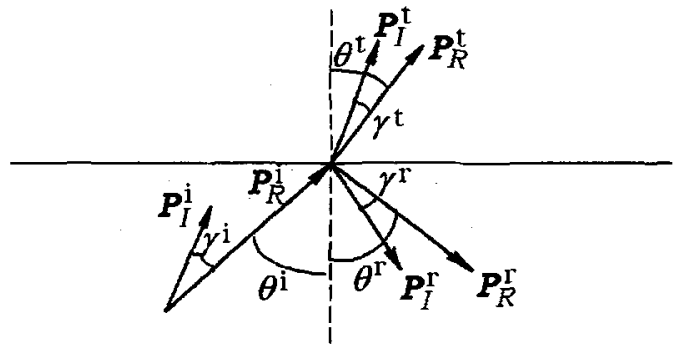

Fig.2 The reflection and refraction of general SH plane wave on perfect interface between two dissimilar viscoelastic bodies

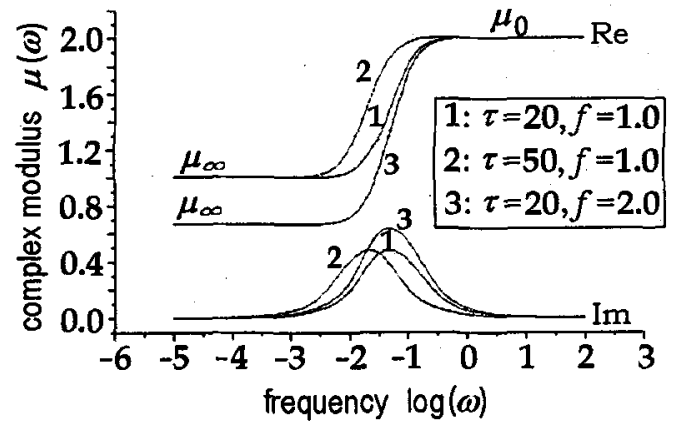

Fig.3 Viscoelastic material complex modulus $\mu(\omega)$ versus $\omega$

Comparing curves 1 and 2 in Fig.3, it can be seen that the relaxation time $\tau$ only affects the location of the frequency influence region, but has no effect on the complex module's peak value. On the other hand, comparing the curves 1 and 3 in Fig.3, it can be seen that the change of viscoelastic parameter $f$ results in the change of complex module's peak value, but with no change on the location of the frequency influence region.

Figure 4 shows the crack opening displacement COD along the crack length for various incident angles $\theta^{\mathrm{i}}$ when $\omega=1$. The numerical results in the figure have been normalized by the COD at the center of the crack. It can be seen that the peak value of COD reaches maximum at $\theta^{\mathrm{i}}=0$ and decreases gradually as the incident angle increases. When the incident angle becomes $90^{\circ}$, the crack surface will close completely. The peak value of COD located at the center of the crack at normal incident and the location is nearly constant as the incident angle increases.
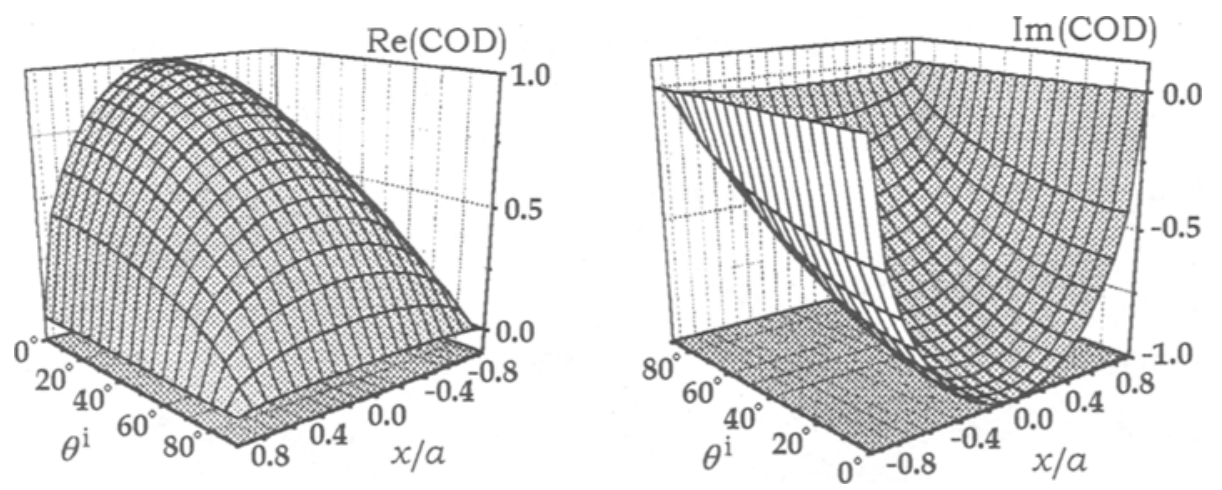

Fig.4 Crack open displacement COD along crack length versus incident angle $\theta^{\mathrm{i}}$ for $\omega=1$

Figure 5 shows the crack opening displacement COD along the crack length for various normal incident frequencies. The numerical results in the figure have been normalized by dividing by the COD for $\omega=1$. It can be seen that the peak value of COD reaches maximum at $\omega=1$ and decreases gradually as the incident frequency decreases. Like that in Fig.4, the peak value of COD always locates at the center of the crack.

Figure 6 shows the dynamics stress intensity factor $K_{\text {III }}$ for various incident angles and incident frequencies. The numerical results in the figure have been normalized by dividing by the dynamic stress intensity factor at $\theta^{\mathbf{i}}=0$ and $\omega=1$. It can be seen that the dynamic stress intensity factor $K_{\mathrm{III}}$ reaches maximum at $\theta^{\mathrm{i}}=0$ and decreases monotonically as the 

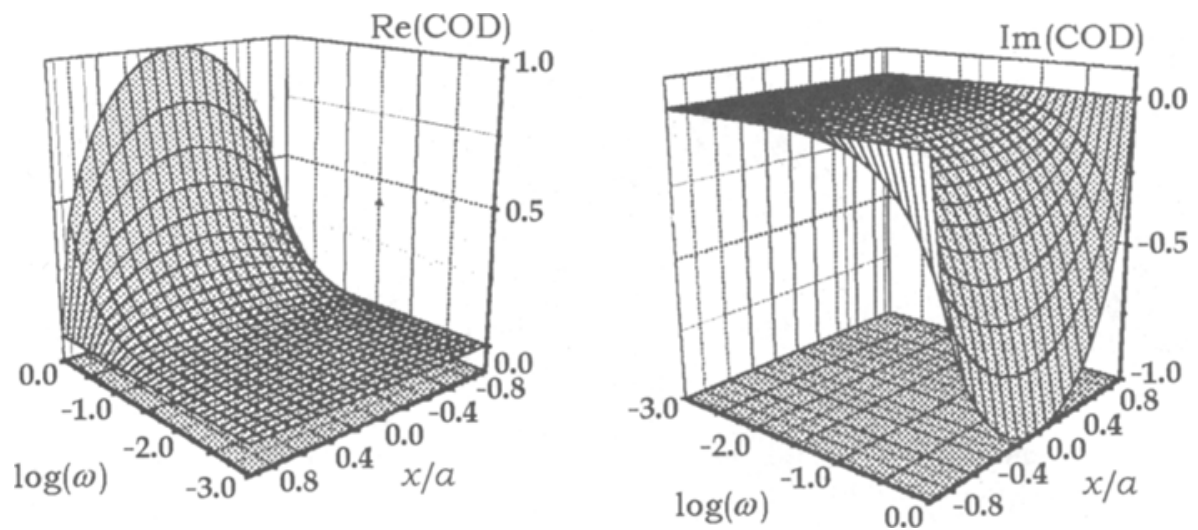

Fig.5 Crack open displacement COD versus frequency $\omega$ for normal incident
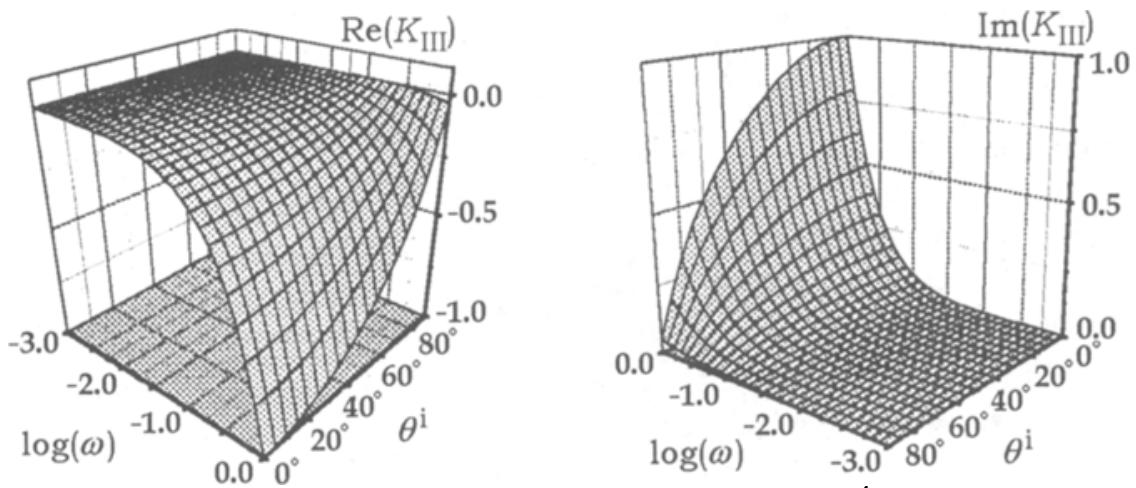

Fig.6 Dynamic stress intensity factor $K_{\text {III }}$ versus incident angle $\theta^{\mathrm{i}}$ and incident frequency $\omega$

incident angle increases when the incident frequency $\omega$ is fixed. For graze incident, $K_{\text {III }}$ is equal to zero. When the incident angle $\theta^{i}$ is fixed, $\left|K_{\text {III }}\right|$ increases monotonically when the frequency $\omega$ increases.

Figure 7 shows the COD for various incident angles $\theta^{i}$ when the dimensionless wave
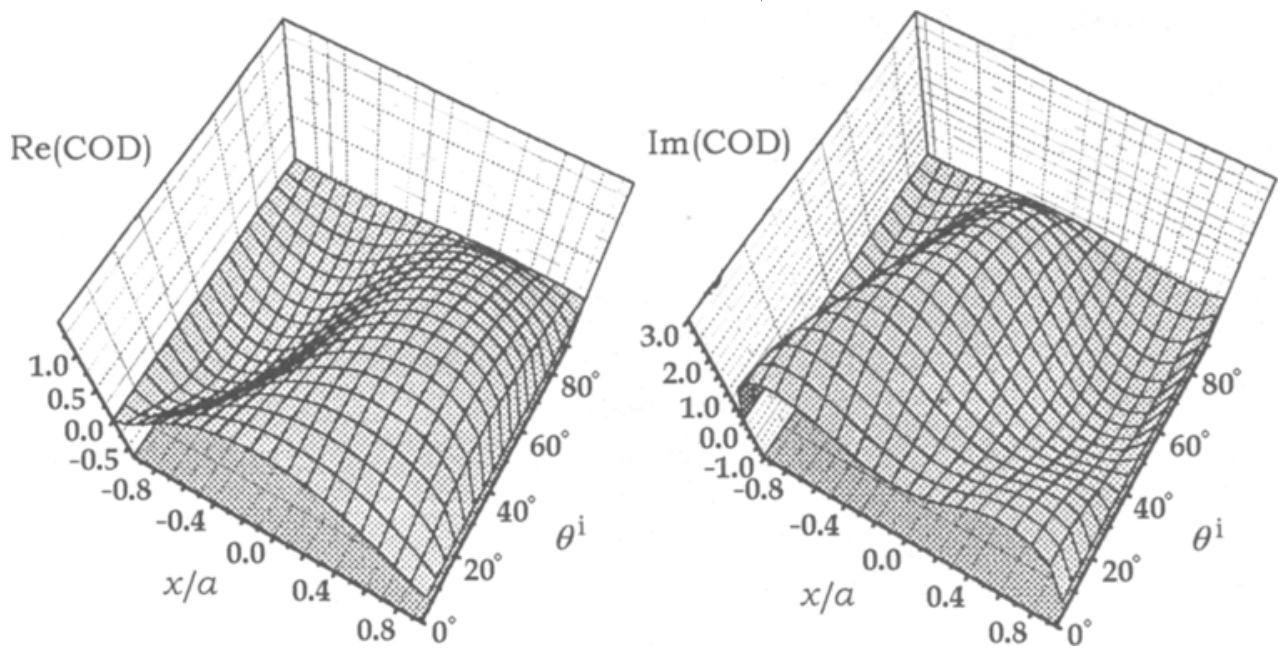

Fig.7 Crack open displacement COD along crack length versus incident angle $\theta^{\mathrm{i}}$ for the case of dimensionless wave number $k=3$ 
Vol.15, No.3 Wei Peijun et al.: Scattering of General SH Plane Wave by Interface Crack 253

number $k=\omega a / \sqrt{\mu_{0} / \rho}=3$. From the figure, it can be seen that the location of the peak value of COD shifts gradually with increasing incident angle, when incident wave length is quantitatively close to crack length.

In summarizing our numerical study, the following conclusions are drawn:

(1) The viscoelastic behavior of materials has effects on the incident wave in the frequency influence region. The region is determined completely by the viscoelastic material parameters: the relaxation time $\tau$ affects the location of the frequency influence region, and $f$ affects the peak value of the complex modulus in the frequency influence region.

(2) For incident wave whose frequency is outside of the frequency influence region, the dynamic response characteristics of viscoelastic materials are equivalent to those of elastic materials with shear modulus $\mu_{\infty}$ for small $\omega$ or those of elastic material with shear modulus $\mu_{0}$ for large $\omega$.

(3) For incident wave whose frequency is within the frequency influence region, when the incident angle $\theta^{\mathrm{i}}$ is fixed, the crack opening displacement COD and the dynamic stress intensity factor $\left|K_{\mathrm{III}}\right|$ increase monotonically as the frequency $\omega$ increases. When the frequency $\omega$ is fixed, they decrease monotonically as the incident angle $\theta^{\mathbf{i}}$ increases and are equal to 0 at $\theta^{\mathrm{i}}=90^{\circ}$.

\section{REFERENCES}

1 Miklowitz J. The Theory of Elastic Wave and Waveguides. New York: North-Holland Publishing Company, 1978

2 Achenbach JD. Wave Propagation in Elastic Bodies. Amsterdam: North-Holland Publishing Company, 1973

3 Srivastava KN, Gupta OP, Palaiya RM. Interaction of elastic waves in two bounded dissimilar elastic half-planes having Griffith crack at interface-I. Int J Frac, 1978, 14: 145 154

4 Srivastava KN, Palaiya RM, Gupta OP. Interaction of longitudinal wave with penny-shaped crack at interface of two bounded dissimiliar elastic bodies-II. Int $J$ Frac, 1979, 15: 591 599

5 Yang HJ, Body DB. Elastic wave scattering from an interface crack in a layered half-space. $J$ Appl Mech, 1985, 52: 42 50

6 Bostrom A. Elastic scattering from an interface crack: Antiplane strain. J Appl Mech, 1987, .54: $503 \sim 508$

7 Ma Xingrui, Zou Zhenzhu, Shao Chengxun, Huang Wenhu. Investigation on the scattering of elastic waves (P and SV) by a plane interface crack in layered media. Acta Aeronautic et Astronautica Sinica, 1989, 10: A434 A441 (in Chinese)

8 Zhang Zimao, Ma Xingrui, Zou Zhenzhu, Wang Duo. Scattering of elastic waves by multiple non-coplane interface Griffith cracks in a layered media. Acta Mechanica Sinica, 1990, 22: $700 \sim 716$ (in Chinese)

9 Qu Jianmin. Interface crack loaded by a time-harmonic plane wave. Int J Solids Struc, 1994, 31: $329 \sim 345$

$10 \mathrm{Qu}$ Jianmin. Scattering of plane waves from an interface crack. Int J Eng Sci, 1995, 33: 179 194

11 Lockett FJ. The reflection and refraction of waves at an interface between viscoelastic materials. J Mech Phys Solids, 1962, 10: 53 63

12 Cooper HF, Reiss EL. Reflection of plane viscoelastic waves from plane boundaries. $J$ Acou Soci America, 1966, 39: 1133 1138

13 Cooper HF. Reflection and transmission of oblique plane waves at a plane interface between viscoelastic media. J Acou Soci America, 1967, 42: 1064 1069 
14 Schoenberg $M$. Transmission and reflection of plane waves at an elastic-viscoelastic interface. Geophys $J$ R Astr Soc, 1971, 25: 35 47

15 Borchert RD. Reflection and refraction of type-II $S$ wave in elastic and anelastic media. Bulletin Seis Soci America, 1977, 67: 43 67

16 Georgiads HG. Plane impact of a cracked viscoelastic body. Int $J$ Eng Sci, 1991, 29: 171 177

17 Geogriads HG. Shear and torsional impact of a cracked viscoelastic body. Int J Solids Struc, 1993, 30: 1891 1906

18 Churchill RV, Brown JW. Fourier Series and Boundary Value Problems. 3rd edn, New York: McGraw-Hill, 1978

19 Muskhelishvili IN. Singular Integral Equations. Groningen: Noordhoff, 1953

20 Erdogan F, Gupta GD, Cook TS. Numerical solution of singular integral equations. In: Sih GC, ed. Mechanics of Fracture V1, Methods of Analysis and Solutions of Crack Problems. Leyden: Noordhoff International Publishing, 1973

21 Kurtz RD, Farris TN, Sun CT. The numerical solution of Cauchy singular integral equations with application to fracture. Int $J$ Fracture, 1994, 66: 139 154 